\title{
Rheological Study on Crosslinking and Gelation of Amidated Carboxymethylcellulose Solutions
}

\author{
R. Lapasin, a," D. Mercuri, ${ }^{b}$ F. Segatti, ${ }^{b}$ G. De Conti, \\ M. Grassi, ${ }^{a}$ and M. Abrami ${ }^{\mathrm{a}}$ \\ aDepartment of Engineering and Architecture, \\ University of Trieste, Trieste, Italy \\ ${ }^{\mathrm{b}} \mathrm{R} \& \mathrm{D}$ Department, Limacorporate, Villanova di San Daniele, Italy
}

doi: 10.15255/CABEQ.2018.1380

Original scientific paper

Received: May 18, 2018

Accepted: November 23, 2018

Viscosupplementation is an intra-articular symptomatic treatment of mild osteoarthritis in synovial joints. It normally consists of single or repeated injections of hyaluronan-based fluids, aimed to restore desirable viscoelastic behavior of the synovial fluid and thus recreate the intra-articular joint homeostasis. Recently, a novel viscosupplement based on amidated carboxymethylcellulose has been developed by blending the soluble polymer (CMCAp) with its crosslinked derivative (CMCAg) in appropriate proportions and concentrations in order to ensure an optimal combination of flow behavior and viscoelastic properties. The present work is concerned with the rheological monitoring of the crosslinking reaction performed at $25{ }^{\circ} \mathrm{C}$ by starting from aqueous CMCAp solutions in order to describe the time evolution of the linear viscoelastic moduli occurring along the whole gelation process and to individuate how the polymer concentration affects the mechanical response at the sol-gel transition and the fractal dimension of the incipient polymeric network.

Keywords:

amidated carboxymethylcellulose, crosslinking, rheology, sol-gel transition, fractal dimension, viscosupplementation

\section{Introduction}

Hydrogels are hydrophilic three-dimensional polymer networks that, in spite of the large amount of water entrapped, display mechanical responses that are, in a certain sense, intermediate between those of liquids and solids. Up to now, numerous hydrogels, often obtained from biopolymers and their derivatives, have been proposed and used in the biomedical field in virtue of their appropriate consistency and biocompatibility ${ }^{1-8}$. Indeed, the rheological properties of the hydrogel-based devices must match the requirements of the various applications, such as scaffolds for tissue engineering, drug delivery systems, cell encapsulation devices and injectable formulations. In the last case both sufficient flowability and proper rheological properties after syringe application must be ensured, in particular fast and substantial structural recovery after shearing, leading to adequate viscoelastic moduli at body temperature ${ }^{-14}$.

Thermoreversible hydrogels can intrinsically ensure these requirements in virtue of the sol-gel transition promoted by heating ${ }^{15-19}$. Poloxamers and other triblock copolymers are low viscosity Newto-

"Corresponding author: Romano.Lapasin@dia.units.it nian liquids at ambient temperature, whereas at sufficiently high polymer concentration they can display a gel behavior and high viscoelastic moduli around physiological temperature. Their sol-gel transition is due to micelle formation and higher order structural organization as micellar cubic gel.

Injectable polymer hydrogels can be also obtained through an adequate degree of chemical crosslinking, apt to ensure proper flowability and viscoelasticity of the system that can then be used as such, or to be employed in the preparation of a polymeric blend. Chemical crosslinking can be a useful approach to overcome some limitations of biomaterials, in particular to provide higher mechanical properties and better stability, under the condition that it does not lead to undesirable effects in terms of degradability and cytotoxicity ${ }^{20-22}$.

This strategy has been adopted also to prepare some supplementation fluids, which are mixtures of linear chain and chemically cross-linked hyaluronic acid (HA), to overcome common treatment issues such as injectability, compromise between gel and liquid behaviour, and prolonging treatment effect $^{23,24}$. Viscosupplementation is an intra-articular symptomatic treatment of mild osteoarthritis (OA) that mainly affects the weight-bearing chondro-synovial joints such as knee, hip and the upper ex- 
tremities $^{25-29}$. Joint performances are closely connected with the viscoelastic properties of synovial fluid, which determines the load transmission and distribution, providing for both lubrication and protection of joint cartilage and soft surfaces against compressive damage. Among other components of the synovial fluid, HA is crucial to the viscoelastic properties that allow the efficient movement of articular joints ${ }^{30,31}$. In OA joint diseases, the concentration and molecular weight of HA decrease and the synovial fluid becomes more abundant, less viscous and reduced in elastoviscous properties that may culminate in a painful condition for the patient. Visco-supplementation normally consists of single or repeated injections of hyaluronan-based fluids, aimed to restore desirable viscoelastic behavior of the synovial fluid and thus to recreate the intra-articular joint homeostasis. One of the most important drawbacks of HA and HA-based derivatives is the multiple injection therapy due to the enzymatic degradation by hyaluronidases in the body. High molecular weight products and cross-linked hydrogels have been developed, but they are able to retard the hyaluronidases attack without preventing it.

The research of other polymers, typically polysaccharides, suitable for viscosupplementation enhances the possibility of hyaluronic acid substitution with other more abundant and cheaper biomaterials. Cellulose-based materials with different structural, mechanical and functional properties have been proposed for several biomedical applications, ranging from tissue engineering to injectable systems ${ }^{32,33}$. Recently, a novel viscosupplement based on amidated carboxymethylcellulose (CMCA) has been developed by blending the soluble polymer with its crosslinked hydrogel derivative in appropriate proportions and concentrations in order to ensure an optimal combination of viscoelastic properties and flow behavior (injectability) together with an acceptable structural recovery after shear ${ }^{34}$.

The present work concerns the rheological monitoring of the crosslinking reaction performed at $25{ }^{\circ} \mathrm{C}$ by starting from aqueous CMCA solutions in order to describe the time evolution of the linear viscoelastic moduli occurring throughout the gelation process and to individuate how the polymer concentration affects the mechanical response at the sol-gel transition and the fractal dimension of the incipient polymeric network. The analysis can also provide other important information from the application point of view, i.e. an estimation of the reaction times needed to obtain a sufficient crosslinking degree and the viscoelastic properties attainable in the final state at different polymer concentration in reacting CMCA solution.

\section{Materials and methods}

\section{Materials}

The raw material is sodium carboxymethylcellulose (average molecular weight: $750 \mathrm{kDa}$, substitution degree 0.8) (CP Kelko). This carboxymethylcellulose (CMC) salt has good solubility in water, and so all the reactions were executed in this solvent. The amidation was carried out in two steps: 1) carboxylate activation with NHS/EDC (N-Hydroxysuccinimide purity $>97 \%$ (Sigma-Aldrich); N-(3-Dimethylaminopropyl)-N'-ethylcarbodiimide hydrochloride purity $>99 \%$ (Actim); 2) quick reaction with Methylamine (40\% water solution, Sigma-Aldrich), molar ratio of 1:1. The amidated CMC is then purified and crosslinked at four different polymer concentrations $c_{p}(1.0,1.4,1.6,1.8 \% \mathrm{wt})$, by using a NHS/EDC solution and 1,3 diaminepropane (purity $99.0 \%$, Sigma-Aldrich) (molar ratio with the polymer $0.5: 1$ ) to obtain a crosslinking degree of about $50 \%$. Due to the high molecular weight of the CMC, it is not possible to achieve a concentration in water greater than $2 \%$. The viscosupplementation product blend has been developed in the manner described previously, using the specific amidated CMC polymer concentration of $1.8 \%$.

\section{Experimental procedure}

The rheological monitoring of the crosslinking process was performed using a rotational controlled stress rheometer (Discovery HR-1, TA Instruments, UK), and a parallel plate geometry (crosshatched surfaces, diameter: $40 \mathrm{~mm}$, gap greater than $2.0 \mathrm{~mm}$ ) with Peltier temperature control. The analysis was carried out at $25 \pm 0.1{ }^{\circ} \mathrm{C}$ and a metal solvent trap was used to prevent solvent (water) evaporation.

After the introduction of crosslinking components, the solution was stirred mechanically for 30 seconds, at room temperature $\left(21 \pm 1{ }^{\circ} \mathrm{C}\right)$ and then $3 \mathrm{~mL}$ of the reacting polymer solution was loaded on the measuring device. The tests started 100-120 seconds after crosslinking agent had been added to the polymer solution. A series of consecutive frequency sweeps (logarithmic sequence of 10 increasing values from 0.025 up to $25 \mathrm{~Hz}$, time needed per sweep: $190 \mathrm{~s}$ ) was carried out at constant stress amplitude $(0.3 \mathrm{~Pa})$ for prolonged time (more than $10000 \mathrm{~s}$ ).

Time-resolved mechanical spectroscopy (TRMS) allows a correct probing of the viscoelastic behavior of transient materials as long as the structural changes occur sufficiently slowly in comparison to the experimental time $\Delta t$, i.e. the period of the oscillatory shear. Accordingly, the shear stress re- 
sponse is almost sinusoidal during $\Delta t$ and correct values of the viscoelastic moduli can still be evaluated. According to the criterion suggested by Mours and Winter ${ }^{35}$, such a condition is largely satisfied in all our experimental tests, even at the lowest frequencies, owing to very small values of the dimensionless mutation numbers $N_{\text {mu }}$ evaluated for both $G^{\prime}$ and $G^{\prime \prime 36}$.

\section{Data analysis}

Isochronous mechanical spectra can be derived from the experimental data obtained from the TRMS tests, as illustrated later. Two linear viscoelastic models were selected to describe the frequency dependence of both moduli since they generally provide a satisfactory quality of fitting for both sol and gel behaviors. They are the generalized Maxwell model (gM) and the fractional Friedrich-Braun model $(\mathrm{FB})^{37}$.

The gM model results from the parallel combination of $N$ Maxwell elements and a purely elastic contribution. Accordingly, the frequency dependence of both moduli is described by the following relationships:

$$
\begin{gathered}
G^{\prime}=G_{e}+\sum_{i=1}^{N} \frac{G_{i} \omega^{2} \lambda_{i}^{2}}{1+\omega^{2} \lambda_{i}^{2}} \\
G^{\prime \prime}=\sum_{i=1}^{N} \frac{G_{i} \omega \lambda_{i}}{1+\omega^{2} \lambda_{i}^{2}}
\end{gathered}
$$

where $G_{i}$ and $\lambda_{i}$ are the relaxation modulus and the corresponding relaxation time of the $i^{\text {th }}$ Maxwell element, respectively, and $G$ is the equilibrium modulus, representing the limiting value of the storage modulus for $\omega 0$. In our cases, it will serve to individuate the plateau modulus which appears at sufficiently long process times in the $G^{\prime}$ profile. In order to reduce the correlation degree between the adjustable parameters, the minimization procedure was performed by adopting the following recurrent constraint for the relaxation times: $\lambda_{i+1}=10 \lambda_{i}$. Actually, the mechanical spectra were described qualitatively well with five Maxwell elements, i.e. with seven adjustable parameters $\left(G_{e}, G_{i}\right.$ and $\left.\lambda_{1}\right)$.

The traces of both moduli can be described, usually with an equivalent fitting quality, by the expressions derived from the FB model, which is based on fractional derivatives of stress and $\operatorname{strain}^{37}$ :

$$
G^{\prime}(\omega)=G_{e}+\Delta G \frac{(\lambda \omega)^{d}\left[\cos \left(d \frac{\pi}{2}\right)+(\lambda \omega)^{c} \cos \left((d-c) \frac{\pi}{2}\right)\right]}{1+2(\lambda \omega)^{c} \cos \left(c \frac{\pi}{2}\right)+(\lambda \omega)^{2 c}}
$$

$$
G^{\prime \prime}(\omega)=\Delta G \frac{(\lambda \omega)^{d}\left[\sin \left(\mathrm{d} \frac{\pi}{2}\right)+(\lambda \omega)^{c} \sin \left((\mathrm{d}-\mathrm{c}) \frac{\pi}{2}\right)\right]}{1+2(\lambda \omega)^{c} \cos \left(\mathrm{c} \frac{\pi}{2}\right)+(\lambda \omega)^{2 c}}
$$

where $\lambda$ is a characteristic relaxation time, $G_{e}$ is the equilibrium modulus, $\Delta G$ is a parameter which rules the magnitude of the fractional viscoelastic contributions, while $c$ and $d$ are related to the fractional orders of the differential operators in the constitutive equation. The FB model gives a parsimonious solution for data fitting since it allows achieving a satisfactory data correlation with only five adjustable parameters.

\section{Results and discussion}

The kinetics of the crosslinking process can be described with good accuracy by the time evolution of the linear viscoelastic moduli determined at the same frequency throughout the series of frequency sweeps. Similar profiles are displayed in a log-log graph, as it can be deduced from the examples reported in Fig. 1, where the kinetics detected at two different polymer concentrations $(1.0 \%$ and $1.8 \%)$ and frequencies $\left(0.628\right.$ and $\left.6.28 \mathrm{rad} \mathrm{s}^{-1}\right)$ are compared.

In almost all cases $G^{\prime}$ is initially lower than $G^{\prime \prime}$ and increases more rapidly in the following times, then passes through a crossover point $\left(G^{\prime}=G^{\prime \prime}\right)$ and eventually tends toward an asymptotic value which is slightly dependent on frequency. As the polymer concentration increases, the elastic character of the system becomes more and more important, so that the crossover condition is attained at higher $G$ values and shorter times. The crossover time $t_{\mathrm{cr}}$ is nearly independent of frequency for the $1.0 \% \mathrm{\% t}$ system, while it slightly decreases with increasing $\omega$ at higher concentrations (see Table 1).

This means that, for these systems also, the crossover condition attained at an arbitrary frequency appears to be an improper criterion to define the sol-gel transition, even if it can provide an approximate evaluation of its time scale. Hereafter, we will examine these arguments in more detail.

As demonstrated in a study dedicated to the kinetics of high methoxyl pectin gelation ${ }^{38}$, the time evolution of both moduli at constant frequency can be described quite satisfactorily with the following sigmoidal expressions:

$$
G(t)=G_{0}+\left(G_{\infty}-G_{0}\right) \frac{\left(t / t_{c}\right)^{m}}{1+\left(t / t_{c}\right)^{m}}
$$

where $G_{0}$ and $G_{\infty}$ are the initial $(t=0)$ and asymptotic $(t \rightarrow \infty)$ values, respectively. Their difference 
(a)

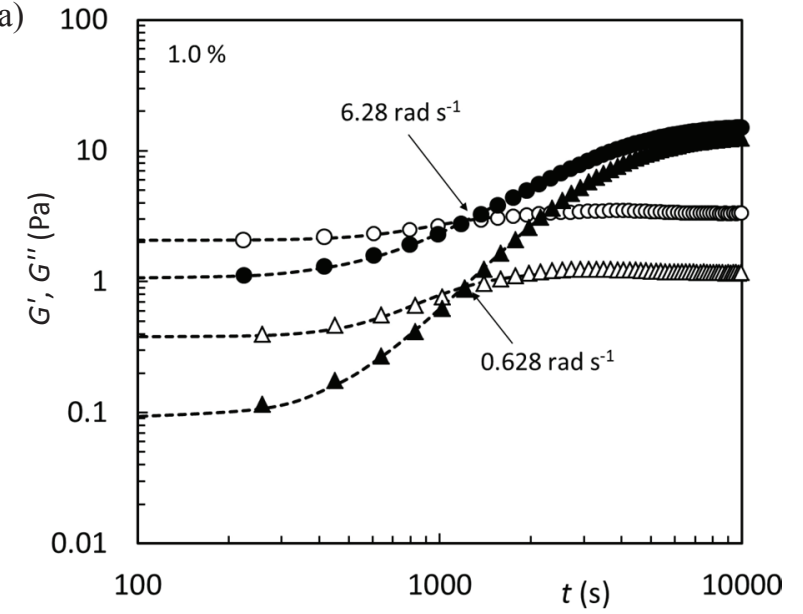

(b)

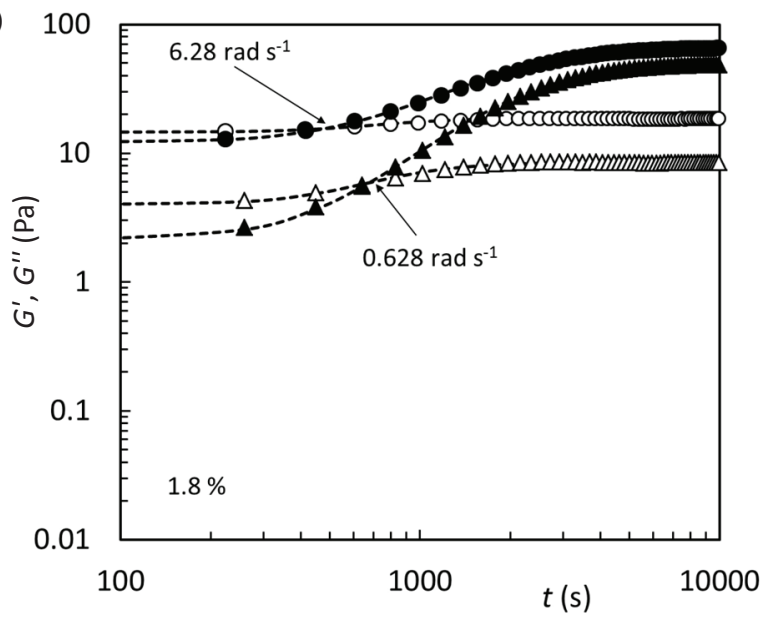

Fig. 1 - Time evolution of the viscoelastic moduli of systems $1.0 \%$ wt (a) and $1.8 \%$ wt (b) at two frequencies. Symbols: $\omega=0.628$ rad $\mathrm{s}^{-1}$ (triangles), $\omega=6.28 \mathrm{rad} \mathrm{s}^{-1}$ (circles); black $\left(G^{\prime}\right)$, white $\left(G^{\prime \prime}\right)$. Lines in the figures represent the best fits of the experimental data obtained with Eq. (3).

$G_{\infty}-G_{0}$ represents the total increase for each modulus throughout the structural process. The characteristic time $t$ corresponds to the mean $G$ value between the two extremes, whereas the exponent $m$ measures the rate of modulus increase. At all frequencies and concentrations the $G^{\prime}$ increase is characterized by longer times $t$ and smaller exponents $m$ in comparison to $G^{\prime \prime}\left(t^{\prime} / t^{\prime \prime}=2.6 \pm 0.4, m^{\prime} / m^{\prime \prime}=\right.$ $0.9 \pm 0.2)$. The individual values of the parameters $\left(t_{c}^{\prime}, t^{\prime \prime}{ }_{c}, m^{\prime}, m^{\prime \prime}\right)$ obtained from data fitting are reported in Appendix (see Table A).

The mechanical spectra derived from the initial and asymptotic values of both moduli can be used to illustrate the maximum change in the linear viscoelastic behavior occurring during the entire course of the crosslinking process, as well as to individuate the transition from sol to gel behavior of all the CMCA systems examined. Fig. 2 reports the initial and final mechanical spectra of the systems $1.0 \%$ and $1.8 \%$, respectively. In both cases the final $G^{\prime}$ curve shows a plateau in the low frequency region, where the storage modulus tends toward an asymptotic value, the equilibrium modulus $G_{e}$ which characterizes the gel character of the CMCA system at the completion of the crosslinking process. Its value can be obtained from data fitting to the selected viscoelastic models with almost the same numerical results, as illustrated hereinafter. All these considerations are obviously extended to the other two systems at intermediate concentrations.

Equation (3) can be profitably used to calculate the $G^{\prime}$ and $G^{\prime \prime}$ values at any time and frequency. Accordingly, the time evolution of the linear viscoelastic behavior in the course of the crosslinking process can be analyzed more thoroughly by comparing the 'isochronous' mechanical spectra calculated from Eq. (3) with the generalized Maxwell model (gM) and the fractional Friedrich-Braun model (FB). Figs. 2a and 2b illustrate the good quality of data fitting with the FB model for the initial and final mechanical spectra of systems $1.0 \% \mathrm{wt}$ and $1.8 \% \mathrm{wt}$, respectively. The generalized Maxwell model generally provides an equivalent quality of fitting for all the systems and process times considered. Fig. 3 reports the mechanical spectra of systems $1.4 \%$ wt (a) and $1.6 \%$ wt (b) calculated at different process times and shows the satisfactory data correlation obtained with the $\mathrm{gM}$ model.

In the case of the $\mathrm{gM}$ model, the transition to the gel behavior is highlighted by the appearance of a purely elastic contribution $G$ beyond a critical gel time and by the parallel evolution of the relaxation time spectrum towards a wedge-box type, due to the

Table 1 -Values of the crossover times $t_{c r}$ (in seconds) obtained from the time evolution of the viscoelastic moduli at different frequencies and concentrations

\begin{tabular}{c|cccccccccc}
\hline \multirow{2}{*}{$c(\% \mathrm{wt})$} & \multicolumn{10}{c}{$\omega\left(\mathrm{rad} \mathrm{s}^{-1}\right)$} \\
\cline { 2 - 23 } & 62.8 & 31.4 & 15.7 & 6.28 & 3.14 & 1.57 & 0.628 & 0.314 & 0.157 \\
\hline 1 & 2256 & 1195 & 1179 & 1222 & 1216 & 1217 & 1195 & 1186 & 1186 \\
1.4 & & 1006 & 1081 & 1107 & 1148 & 1162 & 1176 & 1190 & 1216 \\
1.6 & & & 354 & 354 & 645 & 687 & 714 & 725 & 687 \\
1.8 & & & 478 & 571 & 630 & 677 & 700 & 718 \\
\hline
\end{tabular}


(a)

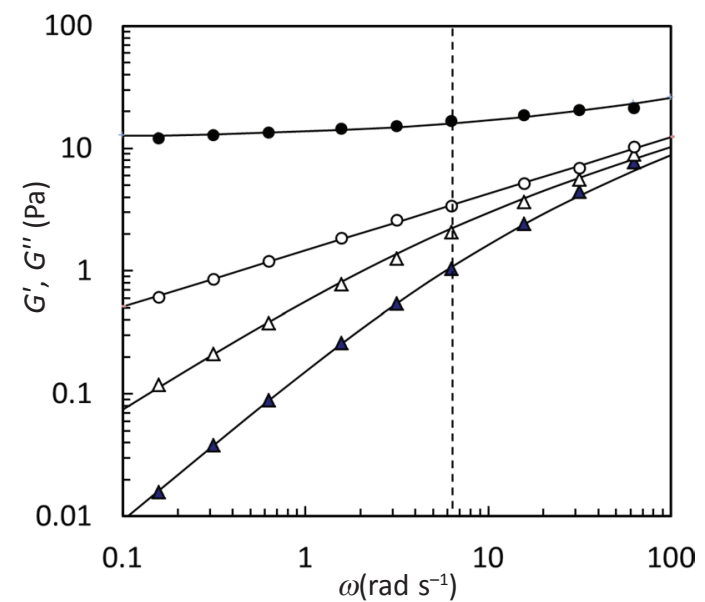

(b)

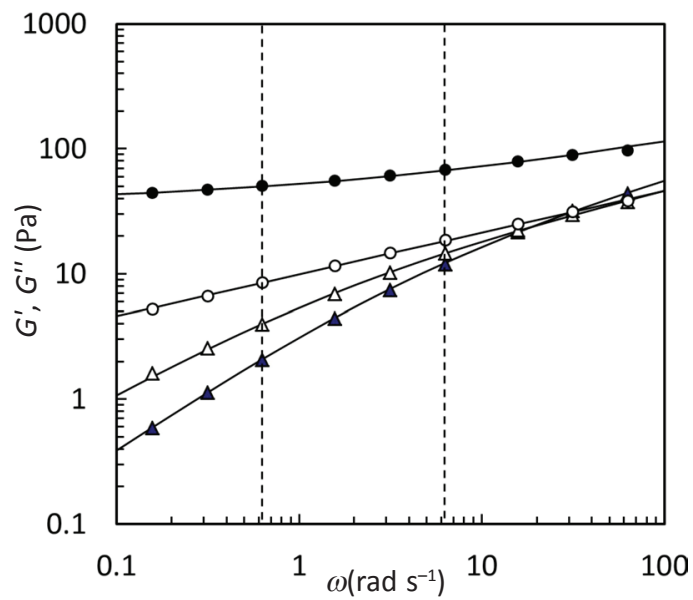

Fig. 2 - Mechanical spectra of systems $1.0 \%$ wt (a) and $1.8 \% w t(b)$ in the initial and asymptotic conditions. Symbols: for $t \rightarrow 0$ (triangles), for $t \rightarrow \infty$ (circles); black $\left(G^{\prime}\right)$, white $\left(G^{\prime \prime}\right)$. Lines in the figures represent the best fits of the experimental data obtained with the FB model (Eqs. (2') and (2')). The dotted lines individuate the frequencies used in the time series depicted in Fig. 1.

(a)

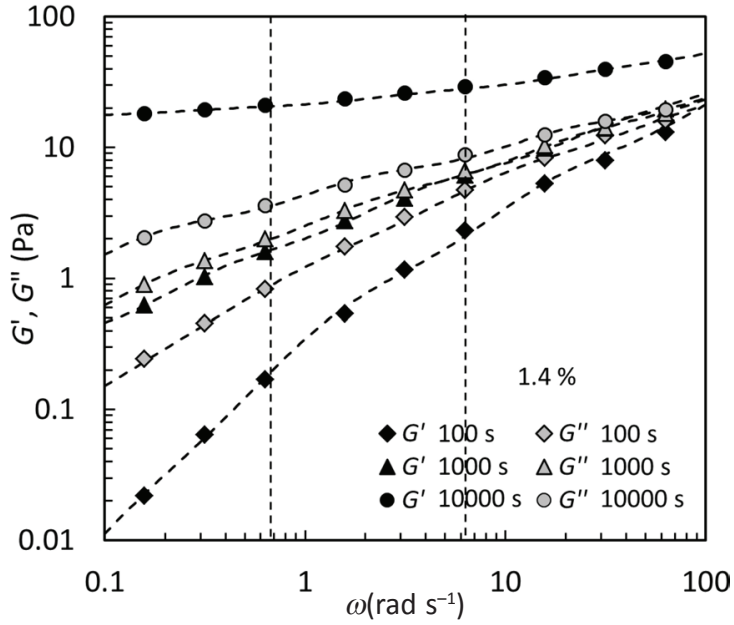

(b)

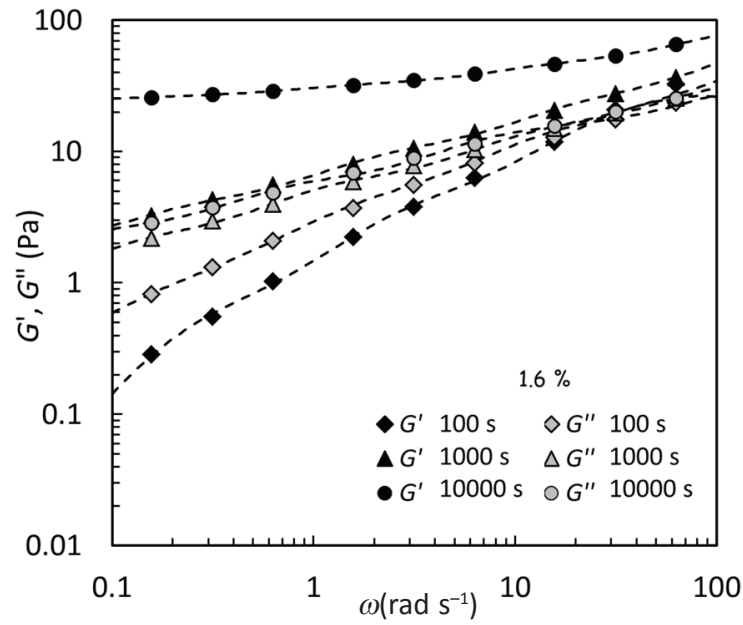

Fig. 3 - Mechanical spectra of systems $1.4 \% w t$ (a) and $1.6 \%$ wt (b) calculated at different process times (in seconds): 100 (diamonds), 1000 (triangles), 10000 (circles), black symbols $\left(G^{\prime}\right)$, grey symbols $\left(G^{\prime \prime}\right)$. Lines in the figures represent the best fits of the experimental data obtained with the gM model (Eqs. (1') and (1"')).

increasing contribution of the longer relaxation modes (see Fig. 4). Such an evolution becomes less pronounced with increasing polymer concentration. The time evolution of $G$ is quite similar for all the systems examined, so that the increasing profiles can be almost superimposed through a double shifting along the two coordinate axes.

In the case of the fractional FB model, the increase in the elastic character due to polymer crosslinking is evidenced by the increasing profile of $G$ vs $t$ (quite similar to that obtained from $\mathrm{gM}$ model) accompanied by the increase in the relaxation time $\lambda$. Moreover, the parallel decrease in parameters $c$ and $d$, which are related to the fractional derivative orders of the model, gives a measure of the progressive transition from viscous to elastic behavior.

Turning to crossover times, their frequency dependence confirms that the criterion proposed by
Tung and Dynes ${ }^{39}$ cannot be used to determine the gel point and other criteria must be invoked ${ }^{40}$. According to Winter and Chambon ${ }^{41-43}$, the sol-gel transition is derived from the common intersection of the $\tan \delta$ vs $t$ curves calculated at different $\omega$. Correspondingly, the traces of both moduli are parallel straight lines in the log-log plot and display a power-law behavior over an extended frequency interval (at least two or more decades). The critical gel state is characterized by the power law relaxation modulus:

$$
G(t)=S t^{-n} \quad \text { for } t>\lambda_{0}, \quad 0<n<1
$$

where $G(t)$ is the relaxation modulus, $S$ is the strength of the critical gel network, $n$ is the relaxation exponent and $\lambda_{0}$ is a lower cutoff time due to the glassy response or entanglement relaxation at small scales ${ }^{44}$. Thus, only two material parameters 

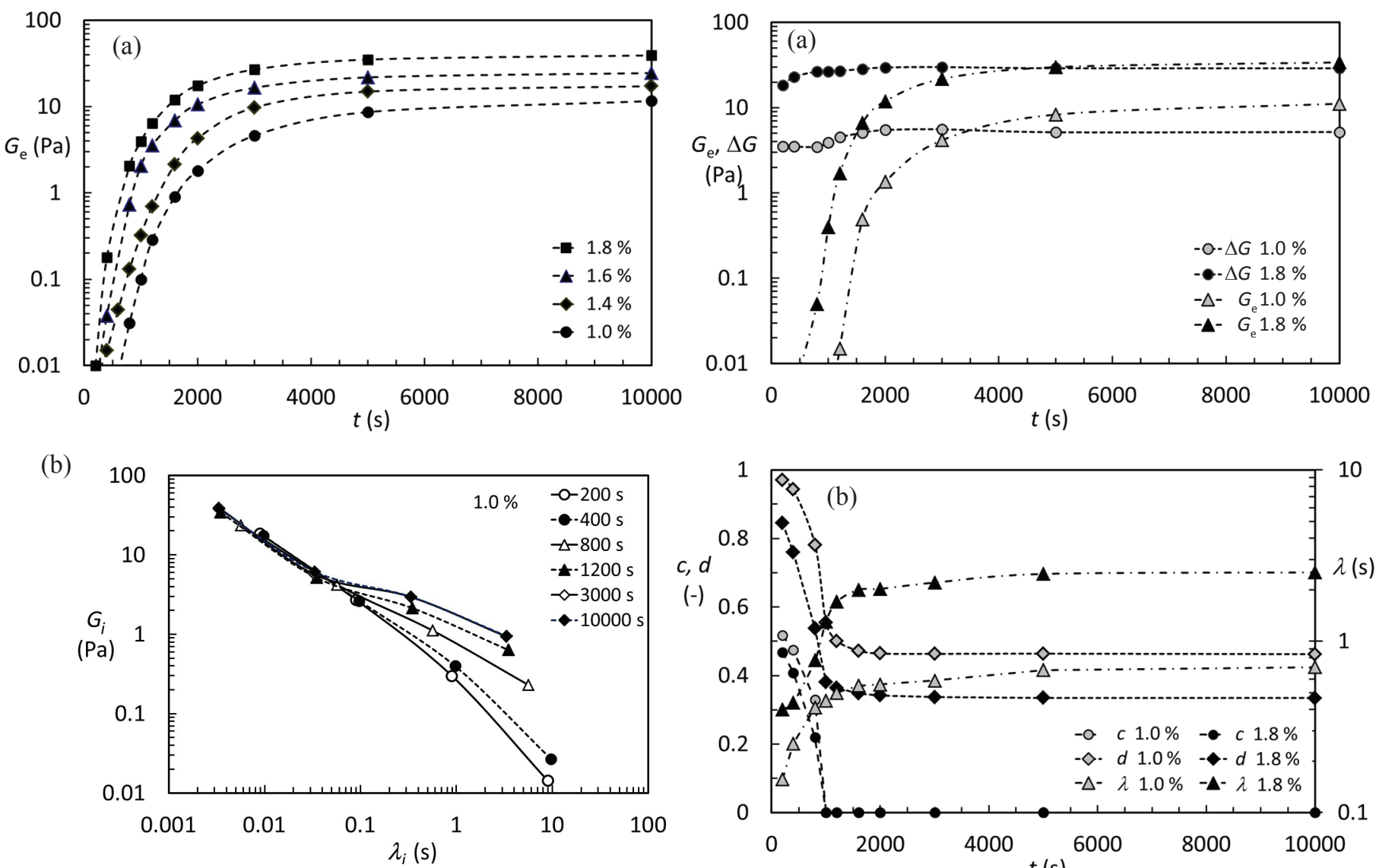

(c)

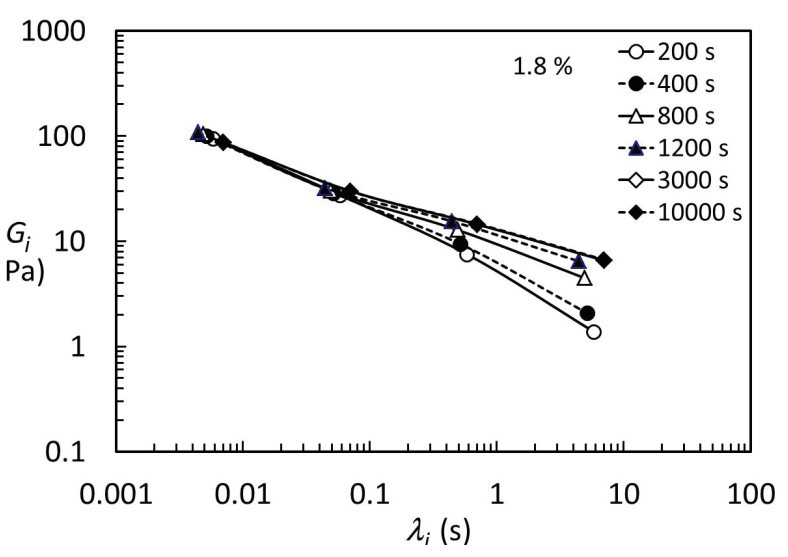

Fig. 4 - Time evolution of the gM parameters. a) Equilibrium modulus $G_{e}$ vs process time at different CMCA concentrations (1.0\%wt (circles), $1.4 \% w t$ (diamonds), $1.6 \% w t$ (triangles), $1.8 \%$ wt (squares); b) time relaxation spectra of systems $1.0 \%$ wt at different process times (in seconds): 200 (orange), 400 (red), 800 (fuchsia), 1200 (violet), 3000 (light blue), 10000 (dark blue); c) time relaxation spectra of systems $1.8 \% \mathrm{wt}$ at different process times (in seconds): 200 (orange), 400 (red), 800 (fuchsia), 1200 (violet), 3000 (light blue), 10000 (dark blue).

are necessary to completely characterize the linear viscoelastic properties of the incipient gel during the crosslinking process. The storage and loss moduli are obtained from the time-dependent modulus via a Fourier transform and, consequently, an analogous power law dependence between dynamic moduli and frequency is derived:

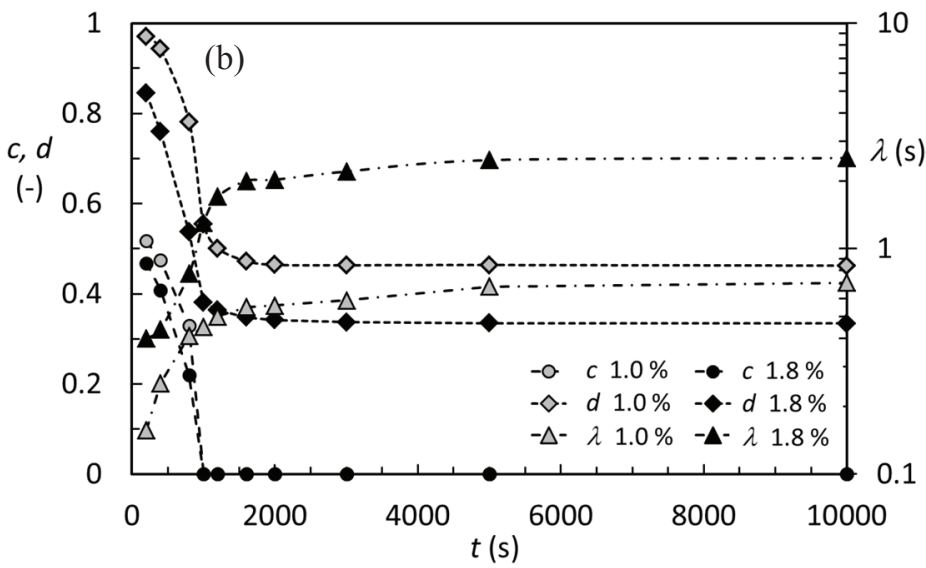

Fig. 5 - Time evolution of the FB parameters for systems 1.0 $\%$ wt (grey symbols) and $1.8 \%$ wt (black symbols). a) Equilibrium modulus $G_{o}$ (triangles) and modulus increment $\Delta G$ (circles); b) c (circles), $d$ (diamonds) and $\lambda$ (triangles).

$$
G^{\prime}=G^{\prime \prime} / \tan \delta=S \omega^{n} \Gamma(1-n) \cos \delta
$$

where $\Gamma(1-n)$ is the Legendre $G$ function. The phase angle $\delta$ between stress and strain is independent of frequency and proportional to the relaxation exponent $n$ :

$$
\delta=\frac{n \pi}{2} \quad \text { or } \quad \tan \delta=G^{\prime \prime} / G^{\prime}=\tan \left(\frac{n \pi}{2}\right)
$$

The Winter-Chambon criterion is satisfied, as may be seen in Figs. 6 and 7. The curves of $\tan \delta$ show a common crossover point that marks the solgel transition. Fig. 7a compares the mechanical spectra of the incipient gel at the different concentrations examined. Both the values and the relative distance of the two moduli increase, thus marking the increase in the elastic character of the system with increasing polymer concentration. Fig. $7 \mathrm{~b}$ shows the corresponding exponential decrease in the loss tangent and the slight decrease in the crossover time $t_{\text {cr }}$.

As polymer concentration increases, the gel stiffness $S$ increases exponentially, while the scaling exponent $n$ linearly decreases (see Fig. 8). 


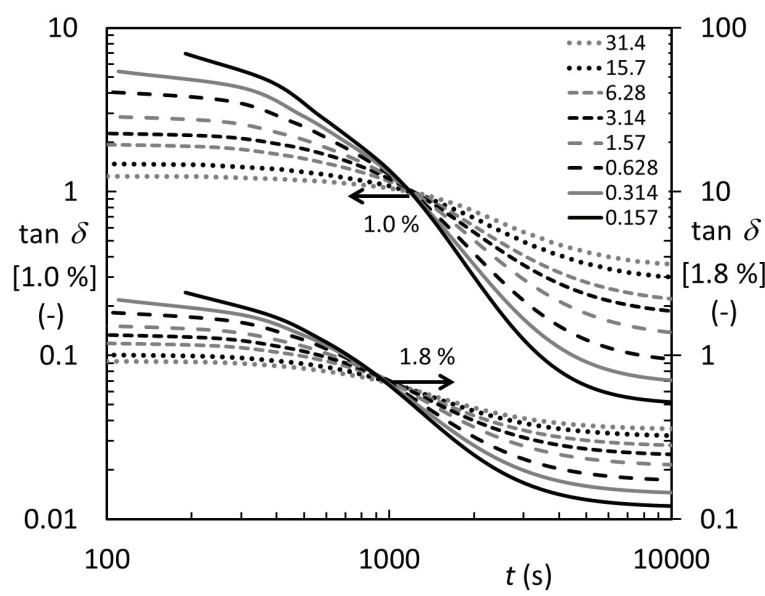

Fig. 6 - Time evolution of the loss tangent at eight different frequencies (from $0.157 \mathrm{rad} \mathrm{s}^{-1}$ to $31.4 \mathrm{rad} \mathrm{s}^{-1}$ ) for system 1.0 $\%$ wt and $1.8 \%$ wt

(a)

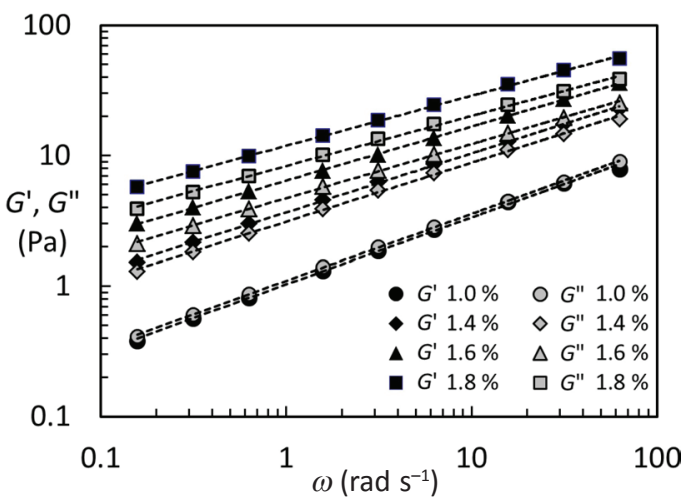

(b)

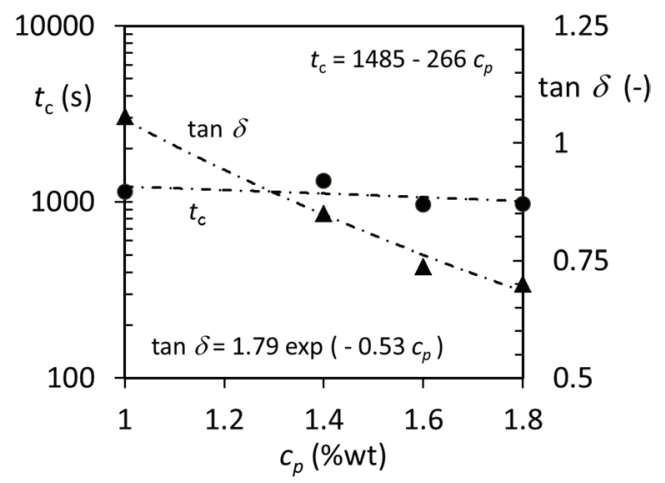

Fig. 7 - a) Mechanical spectra at sol-gel transition. Symbols: $1.0 \% w t$ (circles), $1.4 \% w t$ (triangles), $1.6 \% w t$ (diamonds), and $1.8 \%$ wt (squares); $G^{\prime}$ (black) $G^{\prime \prime}$ (grey) b) concentration dependence of the loss tangent and the crossover time $t_{c r}$ obtained from the application of the Winter-Chambon criterion.

The power-law dependence of the dynamic moduli at the gel point has led to theories suggesting that the crosslinking clusters at the gel point are self-similar and may be described in terms of the fractal geometry in the length scales between the monomer size and the correlation length of connectivity. Accordingly, the fractal dimension $d_{f}$ rules the proportionality between the radius of gyration $R_{g}$ and the mass $M$ of the polymer cluster, i.e. $R_{g}^{g} \sim M^{1 / d_{f}}$. Several theoretical relationships between the relaxation exponent $n$ and the fractal dimension $d_{f}$ of the incipient gel network have been proposed ${ }^{45-47}$. Differences are mainly based on whether the excluded volume of the polymer chains is screened or unscreened near the gel point and polydispersity of crosslinked clusters is accounted for or not. For the case of both polydispersity and fully screened excluded volume effects ${ }^{47}$, the relaxation exponent $n$ can be related to the fractal dimension, $d_{f}$, at the gel point by:

$$
n=\frac{D\left(D+2-2 d_{f}\right)}{2\left(D+2-d_{f}\right)}
$$

where $D$ is the space dimension (i.e., 3). Using this equation, $d_{f}$ values were calculated for our systems. As polymer concentration increases from 1 to $1.8 \%$, the fractal dimension of the incipient gel linearly increases from 1.99 to 2.13, indicating increasing compactness of the polymer clusters (see Fig. 9). Indeed, all the systems evolve towards denser structures as crosslinking proceeds past the sol-gel transition.

A final consideration concerns the viscoelastic characteristics of the gelling systems when the crosslinking reaction is completed. Fig. 10 com-

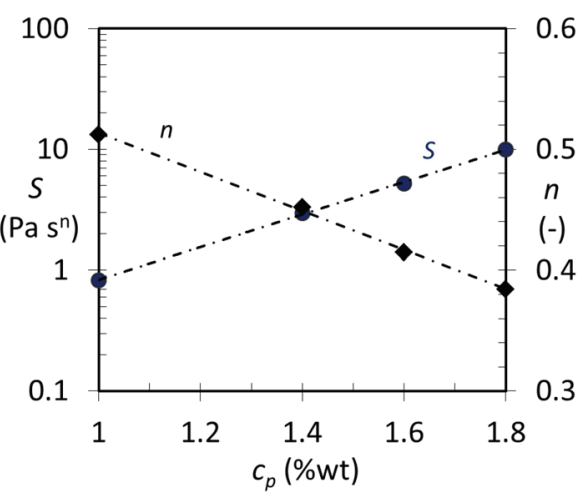

Fig. 8 - Concentration dependence of scaling exponent $n$ (diamonds) and gel strength $S$ (circles)

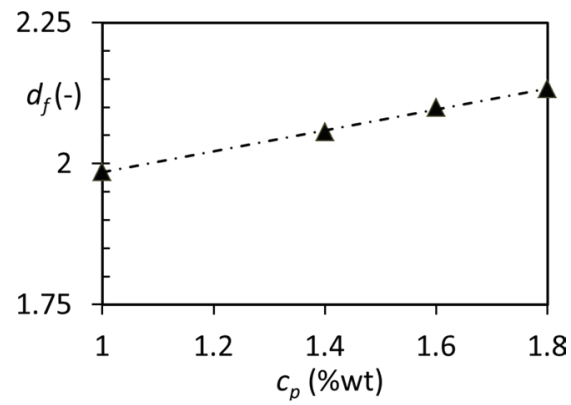

Fig. 9 - Concentration dependence of fractal dimension $d_{f}$ 
(a)

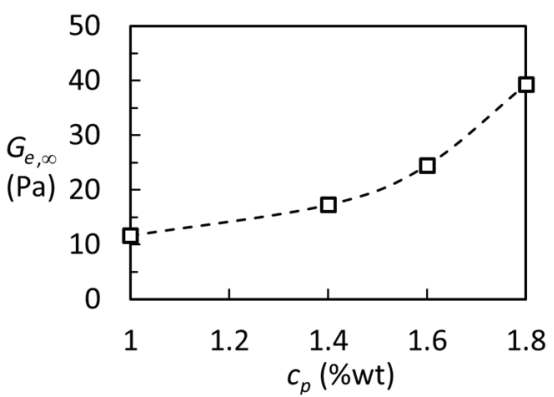

(b)

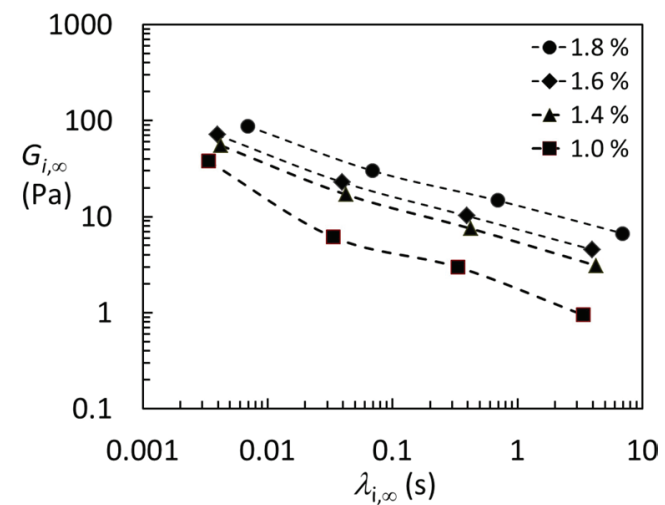

Fig. 10 - Concentration dependence of gM parameters in the final asymptotic condition (for $t \rightarrow \infty$ ). a) Equilibrium modulus $\left.G_{e} b\right)$ time relaxation spectra $G_{i, \infty}-\lambda_{i, \infty}$

pares the GM parameters of the mechanical spectra derived from the asymptotic values, $G_{\infty}^{\prime}$ and $G_{\infty}^{\prime \prime}$, calculated for all the systems., i.e. the equilibrium modulus $G_{e, \infty}$ and the time relaxation spectra given by the $G_{i, \infty}-\lambda_{i, \infty}$ relationships. Both diagrams highlight the increasing elastic character of the gel structure in the final asymptotic state attained at the end of the crosslinking process for increasing concentration.

\section{Conclusions}

The time evolution of the linear viscoelastic moduli in the course of the crosslinking process displays similar characteristics at different frequencies and different polymer concentrations of the reacting CMCA solution. An increase in polymer concentration implies more elastic character of the system, so that the crossover condition is attained at higher $G$ values and shorter times. The sol-gel transition is determined with the Winter-Chambon criterion: such a condition shifts to slightly shorter times with increasing concentration, with linearly decreasing values of the power law relaxation exponent. Correspondingly, the calculated fractal dimension $d_{\text {f }}$ of the polymer clusters linearly increases from 1.99 to 2.13 , thus indicating that the compactness of the incipient gel increases with increasing concentration. Similar $d_{f}$ values and concentration effects have been observed for the critical high acyl gellan gel ${ }^{48}$. The viscoelastic behaviors of the gelling systems in their final asymptotic conditions are quite similar with increasing elastic character for increasing concentration.

\section{A p p e n d i x}

Tab1e A - Values of the time $t_{c}$ and exponent $m$ obtained from data fitting

\begin{tabular}{|c|c|c|c|c|c|c|c|c|c|}
\hline$t_{\mathrm{c}}^{\prime}(\mathrm{s})$ & \multicolumn{9}{|c|}{$\omega\left(\mathrm{rad} \mathrm{s}^{-1}\right)$} \\
\hline$c_{p}(\% \mathrm{Wt})$ & 62.8 & 31.4 & 15.7 & 6.28 & 3.14 & 1.57 & 0.628 & 0.314 & 0.157 \\
\hline 1 & 3219 & 3117 & 3092 & 3268 & 3311 & 3473 & 3530 & 3499 & 3429 \\
\hline 1.4 & 2050 & 2007 & 2101 & 2237 & 2348 & 2460 & 2582 & 2637 & 2650 \\
\hline 1.6 & 1891 & 1806 & 1735 & 1830 & 1909 & 1997 & 2115 & 2192 & 2252 \\
\hline 1.8 & 1718 & 1704 & 1723 & 1781 & 1847 & 1922 & 2028 & 2105 & 2172 \\
\hline$t_{\mathrm{c}}^{\prime \prime}(\mathrm{s})$ & \multicolumn{9}{|c|}{$\omega\left(\operatorname{rad~s}^{-1}\right)$} \\
\hline$c_{p}(\% \mathrm{Wt})$ & 62.8 & 31.4 & 15.7 & 6.28 & 3.14 & 1.57 & 0.628 & 0.314 & 0.157 \\
\hline 1 & 1448 & 1133 & 1081 & 1013 & 1062 & 1013 & 996 & 1000 & 1020 \\
\hline 1.4 & 907 & 1023 & 1012 & 1049 & 1052 & 1086 & 1120 & 1153 & 1196 \\
\hline 1.6 & 631 & 643 & 714 & 744 & 748 & 748 & 753 & 767 & 794 \\
\hline 1.8 & 760 & 772 & 652 & 723 & 734 & 740 & 756 & 775 & 805 \\
\hline
\end{tabular}




\begin{tabular}{|c|c|c|c|c|c|c|c|c|c|}
\hline$m^{\prime}(-)$ & \multicolumn{9}{|c|}{$\omega\left(\operatorname{rad~s}{ }^{-1}\right)$} \\
\hline$c_{p}(\% \mathrm{wt})$ & 62.8 & 31.4 & 15.7 & 6.28 & 3.14 & 1.57 & 0.628 & 0.314 & 0.157 \\
\hline 1 & 4.06 & 2.11 & 1.98 & 2.03 & 2.16 & 2.28 & 2.53 & 2.80 & 3.14 \\
\hline 1.4 & 1.89 & 2.05 & 2.27 & 2.24 & 2.35 & 2.49 & 2.76 & 3.05 & 3.46 \\
\hline 1.6 & 3.20 & 2.29 & 1.97 & 2.00 & 2.04 & 2.10 & 2.22 & 2.35 & 2.53 \\
\hline 1.8 & 2.25 & 2.13 & 2.04 & 2.04 & 2.07 & 2.12 & 2.23 & 2.35 & 2.52 \\
\hline$m^{\prime \prime}(-)$ & \multicolumn{9}{|c|}{$\omega\left(\operatorname{rad~s}{ }^{-1}\right)$} \\
\hline$c_{p}(\% \mathrm{Wt})$ & 62.8 & 31.4 & 15.7 & 6.28 & 3.14 & 1.57 & 0.628 & 0.314 & 0.157 \\
\hline 1 & 10.00 & 4.01 & 3.25 & 3.20 & 2.61 & 3.05 & 3.25 & 3.42 & 3.70 \\
\hline 1.4 & 5.38 & 2.62 & 2.43 & 2.45 & 2.63 & 2.60 & 2.75 & 2.93 & 3.16 \\
\hline 1.6 & 10.00 & 3.77 & 2.81 & 2.61 & 2.58 & 2.62 & 2.66 & 2.75 & 2.90 \\
\hline 1.8 & 10.00 & 6.40 & 3.74 & 2.92 & 2.84 & 2.75 & 2.73 & 2.78 & 2.89 \\
\hline
\end{tabular}

\section{List of symbols}

\section{Latin}

$c, d \quad-$ fractional exponents in Eqs. (2') and $\left(2^{\prime \prime}\right)$, $(-),(0 \leq c \leq d \leq 1)$, corresponding to orders of the fractional derivatives in the constitutive equation ${ }^{37}$

$c_{p} \quad-$ polymer concentration, $\% \mathrm{w}$

D - space dimension, -

$d_{f} \quad-$ fractal dimension, -

$G^{\prime} \quad-$ storage modulus, $\mathrm{Pa}$

$G^{\prime \prime} \quad-$ loss modulus, $\mathrm{Pa}$

$G_{e} \quad-$ equilibrium modulus in Eqs. (1') and (2'), $\mathrm{Pa}$

$G_{e, \approx} \quad-$ equilibrium modulus, $\mathrm{Pa}$

$G_{i} \quad-$ relaxation modulus of the $i^{\text {th }}$ Maxwell element in Eqs. (1') and (1'), Pa

$G_{i, \infty} \quad-$ relaxation modulus of the $i^{\text {th }}$ Maxwell element in the asymptotic time relaxation spectrum $(t \rightarrow \infty), \mathrm{Pa}$

$G_{0} \quad-$ initial values $(t=0)$ of $G^{\prime}\left(G_{0}^{\prime}\right)$ and $G^{\prime \prime}$ $\left(G_{0}^{\prime \prime}\right)$ in Eq. (3), $\mathrm{Pa}$

$G_{\infty} \quad-$ asymptotic values $(t \rightarrow \infty)$ of $G^{\prime}\left(G_{\infty}^{\prime}\right)$ and $G^{\prime \prime}\left(G_{\infty}^{\prime \prime}\right)$ in Eq. (3), Pa

$G(t) \quad-$ relaxation modulus in Eq. (4), $\mathrm{Pa}$

$m \quad-$ exponent for $G^{\prime}\left(m^{\prime}\right)$ and $G^{\prime \prime}\left(m^{\prime \prime}\right)$ in Eq. (3), -

$M \quad-$ mass of the polymer cluster, $\mathrm{Da}$

$n-$ relaxation exponent in Eq. (4), -
$N_{\mathrm{mu}}^{\prime} N_{\mathrm{mu}}^{\prime \prime} \quad-$ dimensionless mutation numbers, $(-)$, defined as $N_{m u}^{\prime}=\frac{2 \pi}{\omega G^{\prime}} \frac{\partial G^{\prime}}{\partial t}, N_{m u}^{\prime \prime}=\frac{2 \pi}{\omega G^{\prime \prime}} \frac{\partial G^{\prime \prime}}{\partial t}$

$R_{g} \quad-$ radius of gyration of the polymer cluster, $\mathrm{nm}$

$S \quad-$ gel stiffness in Eq. (4), $\mathrm{Pa} \mathrm{s}^{\mathrm{n}}$

$t \quad-$ time, s

$t_{c}-$ characteristic times for the time evolution of $G^{\prime}\left(t_{c}^{\prime}\right)$ and $G^{\prime \prime}\left(t_{c}^{\prime \prime}\right)$ in Eq. (3), s

$t_{c r} \quad-$ crossover time, $\mathrm{s}$

$\tan \delta \quad-$ loss tangent, -

\section{Greek}

$\Gamma(1-n)-$ Legendre G function in Eq. (5), -

$\delta \quad-$ phase angle between stress and strain, rad

$\Delta G \quad-$ incremental modulus in Eqs. (2') and (2'), Pa

$\Delta t \quad-$ time period of the oscillatory shear, $\mathrm{s}$, given by $\Delta t=2 \pi / \omega$

$\lambda-$ characteristic relaxation time in Eqs. (2') and $\left(2^{\prime \prime}\right), \mathrm{s}$

$\lambda_{i}-$ relaxation time of the $i^{\text {th }}$ Maxwell element in Eqs. (1') and (1'), s

$\lambda_{i, \infty} \quad-$ relaxation time of the $i^{\text {th }}$ Maxwell element in the asymptotic time relaxation spectrum $(t \rightarrow \infty)$, s

$\lambda_{0} \quad-$ lower cutoff time for validity of Eq. (4), s

$\omega \quad-$ angular frequency, $\operatorname{rad~s}^{-1}$ 


\section{References}

1. Peppas, N. A., Bures, P., Leobandung, W., Ichikawa, H., Hydrogels in pharmaceutical formulations, Eur. J. Pharm. Biopharm. 50 (2000) 276. doi: https://doi.org/10.1016/S0939-6411(00)00090-4

2. Kashyap, N., Kumar, N., Kumar, M. N. V. R., Hydrogels for pharmaceutical and biomedical applications, Crit. Rev. Ther. Drug Carrier Syst. 22 (2005) 107.

doi: https://doi.org/10.1615/CritRevTherDrugCarrierSyst. v22.i2.10

3. Peppas, N. A., Hilt, J. Z., Khademhosseini, A., Langer, R., Hydrogels in biology and medicine: From molecular principles to bionanotechnology, Adv. Mater. 18 (2006) 1345. doi: https://doi.org/10.1002/adma.200501612

4. Slaughter, B. V., Khurshid, S. S., Fisher, O. Z., Khademhosseini, A., Peppas, N. A., Hydrogels in regenerative medicine, Adv. Mater. 21 (2009) 3307. doi: https://doi.org/10.1002/adma.200802106

5. Alhaique, F., Coviello, T., Matricardi, P., Introduction, In Matricardi, P., Alhaique, F. and Coviello, T. (Eds), Polysaccharide Hydrogels: Characterization and Biomedical Applications, Pan Stanford Publishing, Boca Raton, 2015, pp $1-35$.

6. Caló, E., Khutoryanskiy, V. V., Biomedical applications of hydrogels: A review of patents and commercial products, Eur. Polym. J. 65 (2015) 252. doi: https://doi.org/10.1016/j.eurpolymj.2014.11.024

7. Alesa Gyles, D., Diniz Castro, L., Carréra Silva Jr., J. S., Ribeiro-Costa, R. M., A review of the designs and prominent biomedical advances of natural and synthetic hydrogel formulations, Eur. Polym. J. 88 (2017) 373. doi: https://doi.org/10.1016/j.eurpolymj.2017.01.027

8. Ferreira, N. N., Ferreira, L. M. B., Cardoso, V. M. O., Boni, F. I., Souza, A. L. R., Gremião, M. P. D., Recent advances in smart hydrogels for biomedical applications: From self-assembly to functional approaches, Eur. Polym. J. 99 (2018) 117.

doi: https://doi.org/10.1016/j.eurpolymj.2017.12.004

9. Nguyen, M. K., Lee, D. S., Injectable biodegradable hydrogels, Macromol. Biosci. 10 (2010) 563. doi: https://doi.org/10.1002/mabi.200900402

10. Tan, H., Marra, K. G., Injectable, biodegradable hydrogels for tissue engineering applications, Materials 3 (2010) 1746. doi: https://doi.org/10.3390/ma3031746

11. Huynh, C. T., Nguyen, M. K., Lee, D. S., Injectable block copolymer hydrogels: Achievements and future challenges for biomedical applications, Macromolecules 44 (2011) 6629.

doi: https://doi.org/10.1021/ma201261m

12. Li, Y., Rodrigues, J., Tomás, H., Injectable and biodegradable hydrogels: gelation, biodegradation and biomedical applications, Chem. Soc. Rev. 41 (2012) 2193. doi: https://doi.org/10.1039/c1cs15203c

13. Patenaude, M., Smeets, N. M. B., Hoare, T., Designing injectable, covalently cross-linked hydrogels for biomedical applications, Macromol. Rapid Commun. 35 (2014) 598. doi: https://doi.org/10.1002/marc.201300818

14. Ding, X., Wang, Y., Weak bond-based injectable and stimuli responsive hydrogels for biomedical applications, J. Mater. Chem. B 5 (2017) 887. doi: https://doi.org/10.1039/c6tb03052a

15. Jeong, B., Kim, S. W., Bae Y. H., Thermosensitive sol-gel reversible hydrogels, Adv. Drug Deliv. Rev. 54 (2002) 37. doi: https://doi.org/10.1016/j.addr.2012.09.012
16. Klouda, L., Mikos, A. G., Thermoresponsive hydrogels in biomedical applications, Eur. J. Pharm. Biopharm. 68 (2008) 34. doi: https://doi.org/10.1016/j.ejpb.2007.02.025

17. Jeong, B., Kim, S. W., Bae Y. H., Thermosensitive sol-gel reversible hydrogels, Adv. Drug Deliv. Rev. 64 (2012) 154 doi: https://doi.org/10.1016/j.addr.2012.09.012

18. Gong, C., Qi, T., Wei, X., Qu, Y., Wu, Q., Luo, F., Qian, Z., Thermosensitive polymeric hydrogels as drug delivery systems, Curr. Med. Chem. 20 (2013) 79. doi: https://doi.org/10.2174/0929867311302010009

19. Klouda, L., Thermoresponsive hydrogels in biomedical applications: A seven-year update, Eur. J. Pharm. Biopharm. 97 (2015) 338. doi: https://doi.org/10.1016/j.ejpb.2015.05.017

20. Hennink, W. E., Nostrum, C. F., Novel crosslinking methods to design hydrogels, Adv. Drug Deliv. Rev. 64 (2012) 223. doi: https://doi.org/10.1016/j.addr.2012.09.009

21. Martinez, A. W., Caves, J. M., Ravi, S., Li, W., Chaikof, E. $L$., Effects of crosslinking on the mechanical properties, drug release and cytocompatibility of protein polymers, Acta Biomater. 10 (2014) 26. doi: https://doi.org/10.1016/j.actbio.2013.08.029

22. Reddy, N., Reddy, R., Jiang, Q., Crosslinking biopolymers for biomedical applications, Trends Biotechnol. 33 (2015) 362. doi: https://doi.org/10.1016/j.tibtech.2015.03.008

23. Moreland, L. W., Intra-articular hyaluronan (hyaluronic acid) and hylans for the treatment of osteoarthritis: Mechanisms of action, Arthritis Res. Ther. 5 (2003) 54. doi: https://doi.org/10.1186/ar623

24. Avila, L. Z., Gianolio, D. A., Konowicz, P. A., Philbrook, M., Santos, M. R., Miller, R. J., Drug Delivery and Medical Applications of Chemically Modified Hyaluronan, In Garg, H. G., Cowman, M. K. and Hales, C. A. (Eds,), Carbohydrate Chemistry, Biology and Medical Applications, Elsevier Science, Oxford, 2008, pp. 333-357. doi: https://doi.org/10.1016/B978-0-08-054816-6.00015-X

25. Balazs, E. A., Denlinger, J. L., Viscosupplementation: A new concept in the treatment of osteoarthritis, J. Rheumatol. Suppl. 39 (1993) 3.

26. Balazs, E. A., Viscosupplementation for treatment of osteoarthritis: From initial discovery to current status and results, Surg. Technol. Int. 12 (2004) 278.

27. Stitik, T. P., Levy, J. A., Viscosupplementation (Biosupplementation) for osteoarthritis, Am. J. Phys. Med. Rehabil. 85 (Suppl 11) (2006) S32. doi: https://doi.org/10.1097/01.phm.0000245677.20294.c2

28. Rutjes, A. W. S., Jüni, P., da Costa, B. R., Trelle, S., Nüesch, E., Reichenbach, S., Viscosupplementation for osteoarthritis of the knee: A systematic review and meta-analysis, Ann. Intern. Med. 157 (2012) 180. doi: https://doi.org/10.7326/0003-4819-157-3-201208070-00473

29. Legré-Boyer, V., Viscosupplementation: Techniques, indications, results, Orthop. Traumatol. Surg. Res. 101 (2015) S101. doi: https://doi.org/10.1016/j.otsr.2014.07.027

30. Ogston, A. G., Stanier, J. E., The physiological function of hyaluronic acid in synovial fluid; viscous, elastic and lubricant properties, J. Physiol. 119 (1953) 244. doi: https://doi.org/10.1113/jphysiol.1953.sp004842

31. Gigante, A., Callegari, L., The role of intra-articular hyaluronan (Sinovial) in the treatment of osteoarthritis, Rheumatol. Int. 31 (2011) 427. doi: https://doi.org/10.1007/s00296-010-1660-6 
32. Halib, N., Perrone, F., Cemazar, M., Dapas, B., Farra, R., Abrami, M., Chiarappa, G., Forte, G., Zanconati, F., Pozzato, G., Murena, L., Fiotti, N., Lapasin, R., Cansolino, L., Grassi, G., Grassi, M., Potential applications of nanocellulose-containing materials in the biomedical field, Materials 10 (2017) 977.

doi: https://doi.org/10.3390/ma10080977

33. Milcovich, G., Antunes, F. E., Farra, R., Grassi, G., Grassi, M., Asaro, F., Modulating carbohydrate-based hydrogels as viscoelastic lubricant substitute for articular cartilages, Int. J. Biol. Macromol. 102 (2017) 796. doi: https://doi.org/10.1016/j.ijbiomac.2017.04.079

34. Lapasin, R., Segatti, F., Mercuri, D., De Conti, G., Spagnul, C., Fusi, S., Rheological studies dedicated to the development of a novel injectable polymeric blend for viscosupplementation treatment, Chem. Biochem. Eng. Q. 29 (2015) 51. doi: https://doi.org/10.15255/CABEQ.2014.2148

35. Mours, M., Winter, H. H., Time-resolved rheometry, Rheol. Acta 33 (1994) 385. doi: https://doi.org/10.1007/BF00366581

36. Winter, H. H., Morganelli, P., Chambon, F., Stoichiometry effects on rheology of model polyurethanes at the gel point, Macromolecules 21 (1988) 532.

doi: https://doi.org/10.1021/ma00180a048

37. Friedrich, Chr., Braun, H., Generalized Cole-Cole behavior and its rheological relevance, Rheol. Acta 31 (1992) 309.

doi: https://doi.org/10.1007/BF00418328

38. Giacomazza, D., Bulone, D., San Biagio, P. L., Lapasin, R., The complex mechanism of HM pectin self-assembly: A rheological investigation, Carbohydr. Polym. 146 (2016) 181.

doi: https://doi.org/10.1016/j.carbpol.2016.03.046

39. Tung, C.-Y. M., Dynes, P. J., Relationship between viscoelastic properties and gelation in thermosetting systems, J. Appl. Polym. Sci. 27 (1982) 569. doi: https://doi.org/10.1002/app.1982.070270220
40. Lapasin, R., Rheological Characterization of Hydrogels, In Matricardi, P., Alhaique, F. and Coviello, T. (Eds), Polysaccharide Hydrogels: Characterization and Biomedical Applications, Pan Stanford Publishing, Boca Raton, 2015, pp 83-137. doi: https://doi.org/10.1201/b19751-4

41. Chambon, F., Winter, H. H., Stopping of cross-linking reaction in a PDMS polymer at the gel point, Polym. Bull. 13 (1985) 499. doi: https://doi.org/10.1007/BF00263470

42. Winter, H. H., Chambon, F., Analysis of linear viscoelasticity of a crosslinking polymer at the gel point, J. Rheol. 30 (1986) 367. doi: https://doi.org/10.1122/1.549853

43. Chambon, F., Winter, H. H., Linear viscoelasticity at the gel point of a crosslinking PDMS with imbalanced stoichiometry, J. Rheol. 31 (1987) 683. doi: https://doi.org/10.1122/1.549955

44. De Rosa, M. E., Winter, H. H., The effect of entanglements on the rheological behavior of polybutadiene critical gels, Rheol. Acta 33 (1994) 220. doi: https://doi.org/10.1007/BF00437307

45. Winter, H. H., Mours, M., Rheology of polymers near liquid-solid transitions, Adv. Polym. Sci. 134 (1997) 165. doi: https://doi.org/10.1007/3-540-68449-2_3

46. Muthukumar, M., Winter, H. H., Fractal dimension of a cross-linking polymer at the gel point, Macromolecules 19 (1986) 1284 doi: https://doi.org/10.1021/ma00158a064

47. Muthukumar, M., Screening effect on viscoelasticity near the gel point, Macromolecules 22 (1989) 4656. doi: https://doi.org/10.1021/ma00202a050

48. Chen, Q., Ma, H., Yuan, Y., Han, X., Zhu, J., Zhang, H., Rheological behavior of high acyl gellan gum solution at gel, Int. J. Food Prop. 20 (2017), S2332. doi: https://doi.org/10.1080/10942912.2017.1362431 